\title{
The validity of assessing temporal events, sub-phases and trunk kinematics of the sit-to-walk movement in older adults using a single inertial sensor
}

\author{
Stefan Walgaard ${ }^{\mathrm{a}, *}$, Gert S. Faber ${ }^{\mathrm{b}}$, Rob C. van Lummel ${ }^{\mathrm{a}}$, Jaap H. van Dieën ${ }^{\mathrm{b}}$, \\ Idsart Kingma ${ }^{\mathrm{b}}$ \\ a McRoberts, Raamweg 43, 2596 HN The Hague, The Netherlands \\ ${ }^{\mathrm{b}}$ Research Institute MOVE, Department of Human Movement Sciences, VU University Amsterdam, Van der Boechorststraat 9, 1081 BT Amsterdam,
} The Netherlands

\section{A R T I C L E I N F O}

\section{Article history:}

Accepted 10 March 2016

\section{Keywords:}

Inertial sensor

Sit-to-walk

3D motion validity

Accelerometers

Gyroscopes

\begin{abstract}
A B S T R A C T
The aim of this study was to develop a method to identify temporal events, sub-phases and trunk kinematics of the sit-to-walk (STW) using a single inertial sensor (IS) worn at the lower back and to determine the validity of this method. Twenty-seven healthy older adults performed a STW movement, which started from sitting in a chair and included walking $3 \mathrm{~m}$.

Participants' movements were recorded with the IS, a reference measurement system consisting of an optical motion capture system (3 markers on the IS and one on each foot) and on-off switches located in the seat of the chair.

Using the data from the IS and the reference measurement systems, the following signals and variables were calculated and compared: 3D IS motion (accelerations, velocities, displacements and angles), temporal events (start of trunk movement, seat-off, end of trunk flexion phase, end of trunk rising phase and gait initiation) and trunk kinematics (flexion range, maximum flexion velocity, maximum forward velocity and forward velocity during seat-off and at first heel-strike and maximum vertical velocity and vertical velocity at first heel-strike).

For most variables acceptable differences (RMSE $<10 \%$ ) were found between IS and reference measurement systems, except for sideways displacements and non-sagittal plane rotations. Furthermore, good results were found for temporal event detection, with ICC values for all variables being 0.988 or higher. With exception of the vertical velocity at heel-strike agreement for trunk kinematics was high, with ICC values being 0.867 or higher.
\end{abstract}

(c) 2016 Elsevier Ltd. All rights reserved.

\section{Introduction}

Researchers and clinicians usually assess physical functioning of older people through self-report. To assess the ability to perform daily activities in a more objective way, standardized clinical physical performance tests have been developed. A number of these tests are widely used, such as the Timed Up and Go (Podsiadlo and Richardson, 1991) and the Short Physical Performance Battery (Guralnik et al., 1994). These tests include one of the most demanding daily activities: rising from a chair, which is of key importance for independent functioning. Conventionally, manually recorded time events are used as outcome measures.

\footnotetext{
* Corresponding author. Tel.: +31703106462.

E-mail address: s.walgaard@mcroberts.nl (S. Walgaard).
}

More recently, camera-based systems and force plates have been added to explore the kinematics of rising from a chair. Kinematic variables have been found that contribute to identifying people at risk of losing independent functioning (Bernardi et al., 2004; Buckley et al., 2008; Chen and Chou, 2013; Kerr and Kerr, 2001). However these measurement systems are bound to a lab environment and are only to a limited extent used for routine clinical assessment. For routine clinical use, an easy to handle, efficient and unobtrusive measurement system is more suitable.

Inertial sensors (IS) may offer a potential for use in research and clinical practice. A single IS, worn on the lower back, has successfully been used for analyzing sub-phases and trunk kinematics during chair rise (Giansanti and Maccioni, 2006; Millor et al., 2013; Schwenk et al., 2012; Van Lummel et al., 2012; Van Lummel et al., 2013; Weiss et al., 2011, 2010) and have been validated against other instrumentation for the sit-to-stand task (Boonstra et al., 2006; Janssen et al., 2008; Zijlstra et al., 2012). But 
the validity of using an IS during the biomechanically challenging sit-to-walk (STW) task, which is common in daily life, has not yet been investigated (Kouta and Shinkoda, 2008). Therefore, the aim of this study was to develop and validate a method to identify relevant temporal events, sub-phases and trunk kinematics of the STW using a single IS.

\section{Methods}

\subsection{Participants}

Twenty-seven healthy older adults participated in this study (13 female; mean age: $74.7 \pm 8.5$ years; mean weight: $76.8 \pm 13.2 \mathrm{~kg}$; mean height: $172.2 \pm 8.2 \mathrm{~cm}$ ), which had been approved by the ethics committee of the faculty of human movement sciences (ECB 2014-3M). Prior to testing, all participants provided written informed consent.

\subsection{Movement task}

STW started from sitting in a chair without armrests and a seat height of $44.5 \mathrm{~cm}$ and included walking $3 \mathrm{~m}$. STW initiated after a countdown from 5 to 1 followed by a verbal go command. At the end of the STW, participants were asked to stand still. Two tests were performed in which the first one served as a practice trial.

\subsection{Instrumentation}

Participants wore a single IS (DynaPort ${ }^{\mathbb{B}}$ Hybrid, McRoberts) which was inserted in an elastic belt at the level of the fourth lumbar spinous process (Rispens et al., 2014; Zijlstra et al., 2012). The DynaPort included 3 accelerometers and 3 gyroscopes with a sample rate of 100 samples/s.

Motion data were captured with an optical motion capture system (Optotrak Certus $^{\mathbb{R}}$, Northern Digital Inc.) at 200 samples/s and were resampled to 100 samples/s. Two single markers were attached to the heels of the shoes. Furthermore, a cluster of markers was mounted on a metal plate which was attached to the DynaPort. The marker cluster was related to the DynaPort by digitizing specified positions of the DynaPort using a probe with six markers.

The custom-made chair contained a plate on top of the seat, with four on/off switches between plate and seat. DynaPort, Optotrak and switch data were electronically synchronized.

\subsection{STW assessment}

Before data processing, Optotrak (displacements) and DynaPort (accelerations and angular velocities) were filtered with a bi-directional second-order low-pass Butterworth filter at $15 \mathrm{~Hz}$ cut-off frequency. For DynaPort 3D velocities, displacements and angles were calculated. For Optotrak, 3D accelerations, velocities and angles were calculated (Fig. 1). After each differentiation and integration step, the signals were filtered using a filter as described above.

\subsubsection{Optotrak}

At each time instant, an arbitrary technical coordinate system (CS) was constructed based on the position of the markers on the DynaPort. Subsequently, this CS was aligned with the global CS and expressed relative to the sitting posture by post-multiplying the orientation matrix at each time-sample by the inverse of the orientation matrix during the sitting posture (Faber et al., 2013). Euler angles were calculated based on the aligned orientation matrix using the following Euler angle decomposition order: flexion (leftward axis), lateral (forward axis) and axial (upward axis). Linear velocities and linear accelerations were calculated by differentiating and double differentiating the displacements averaged over markers.

\subsubsection{DynaPort}

DynaPort orientations were defined for the sitting and standing postures based on the gravity vector measured by the accelerometers (Moe-Nilssen, 1998). The heading of the DynaPort was assumed to be zero at these instances.

Next, the orientation matrix was rotated over time for each time-sample by integrating the angular velocities. Due to integration drift, the calculated integrated DynaPort orientation matrix at the standing posture was slightly different from the orientation matrix determined based on the accelerations. Using this orientation error, the orientation matrix time series were corrected. To get a smooth curve, the error correction was distributed over the whole movement period (rotating around the helical axis of the error matrix with the identity matrix), assuming that error grows linearly with time.
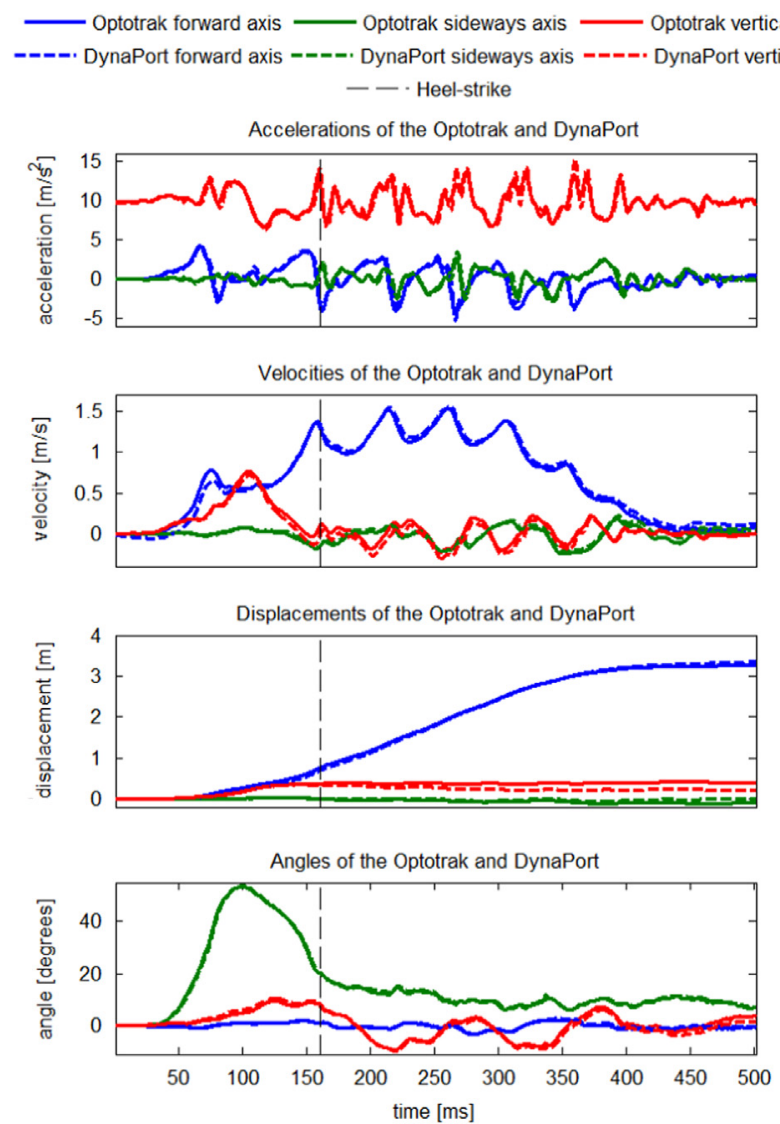

Fig. 1. Example of the 3D accelerations (first pane), velocities (second pane), displacement (third pane) and angles (fourth pane) calculated with the Optotrak and the DynaPort.

Based on the corrected orientation matrix, a new orientation matrix was calculated, describing DynaPort orientation relative to the global axis system, with gravity upward and overall displacement in the horizontal plane defined as forward. Euler angles were calculated, identical to those for the Optotrak described above.

DynaPort accelerations were rotated to the global CS using the DynaPort orientation calculated above. Next, the offset on the accelerations caused by gravity was removed. To obtain velocities, the accelerations were integrated over time. These velocities are subject to integration drift, causing the velocities to be non-zero at the ending posture. To correct for this, the error was linearly distributed over time and subtracted from the velocities between sitting and standing postures. To obtain displacements, the velocities were integrated over time.

Because the DynaPort was not perfectly aligned with the trunk segment and subjects did not sit perfectly straight on the chair, the forward axis of the DynaPort was not pointing perfectly forward in the starting posture. This resulted in a nonstraight trajectory. To correct for this, trajectories, velocities and accelerations were rotated around the vertical such that there was no sideways displacement between the sitting and standing posture.

\subsubsection{Event detection}

Based on accelerations, velocities, trajectories and angles, the following events were defined: start of trunk movement, seat-off, end of trunk flexion phase, end of trunk rising phase and gait initiation. For both Optotrak and DynaPort, start of trunk movement was defined as the start of flexion rotation after the sitting period (Van Lummel et al., 2013) (Fig. 2, pane 1). Using the on-off switches, seat-off was defined as the first instant when all switches were off (Kralj et al., 1990) (Fig. 2, pane 2). Using the DynaPort, seat-off was defined as the minimum vertical acceleration within $0.1 \mathrm{~s}$ of first $25 \%$ of the vertical trajectory range (Fig. 2, pane 3). For both Optotrak and DynaPort end of trunk flexion phase was defined as the instant when the derivative of the flexion rotation was 0 (Fig. 2, pane 4) and end of trunk rising phase was defined as the downward peak vertical velocity after the upward vertical velocity reached zero (Fig. 2, pane 5). Using the Optotrak, gait initiation was defined as first heel-strike: the instant when the derivative of the vertical trajectory of the marker on the stepping leg reached 0 after a local minimum (Fig. 2, pane 6). Using the DynaPort, gait initiation was defined using high frequency components of sensor signals. Raw 

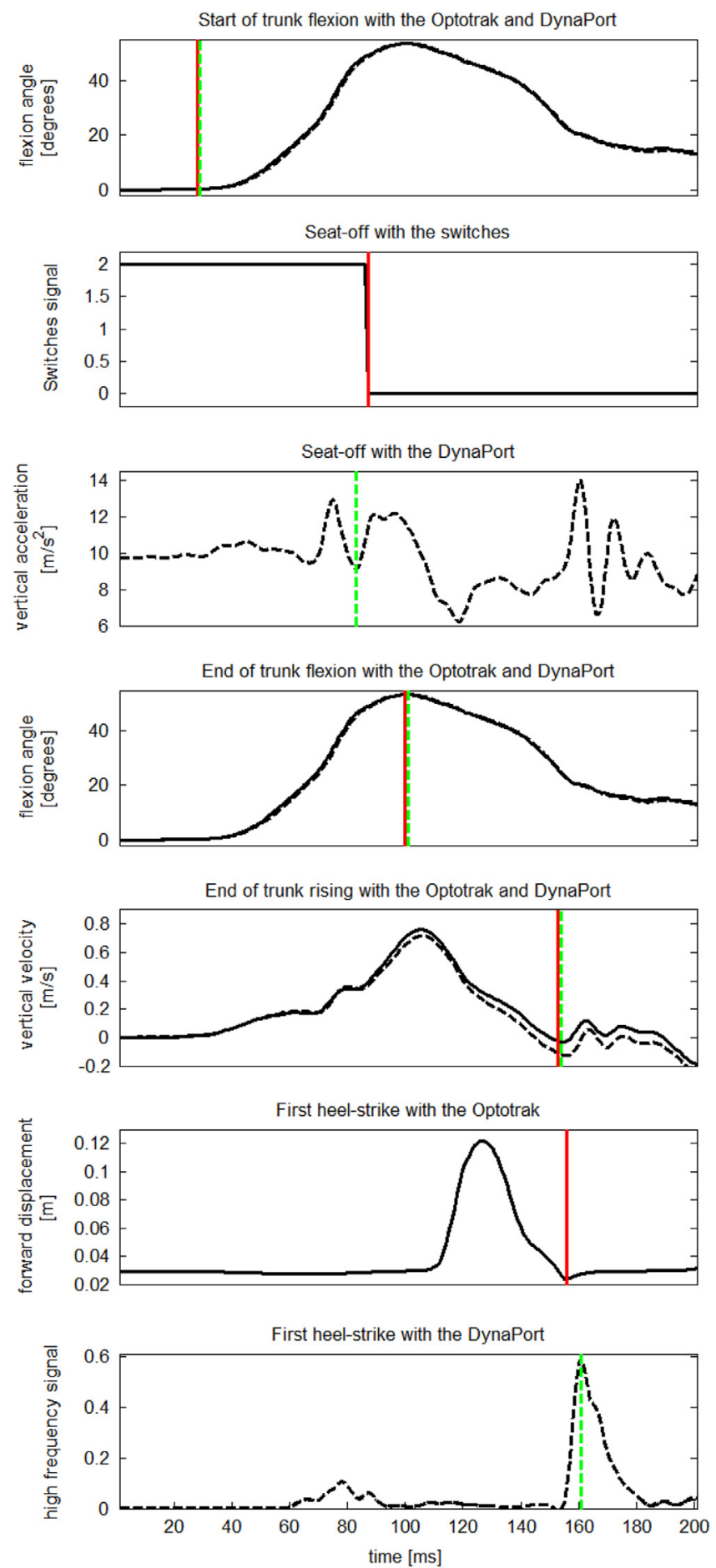

Fig. 2. Example of the start of trunk flexion phase (first pane), seat-off with the switches (second pane), seat-off with the DynaPort (third pane), end of trunk flexion phase (fourth pane), end of trunk rising phase (fifth pane), heel-strike with the Optotrak (sixth pane) and heel-strike with the DynaPort (seventh pane).

accelerations and angular velocities were filtered with a bi-directional secondorder high-pass Butterworth filter at $30 \mathrm{~Hz}$ cut-off frequency. The absolutes of these signals were normalized, summed and subsequently low-pass filtered with a second-order Butterworth filter at $10 \mathrm{~Hz}$ cut-off frequency. Gait initiation was defined as the first peak above the mean signal value after end of trunk flexion phase (Fig. 2, pane 7).

The following trunk kinematics were defined: flexion range, maximum flexion velocity, maximum forward velocity and forward velocity during seat-off and at first heel-strike and maximum vertical velocity and vertical velocity at first heelstrike (Bernardi et al., 2004; Buckley et al., 2008; Chen and Chou, 2013; Kouta and Shinkoda, 2008; Papa and Cappozzo, 2000; Van Lummel et al., 2013).
2.5. Statistics

The consistency between DynaPort and Optotrak signals was compared for trunk angular and linear motions using the root mean square of the error (RMSE) and the RMSE relative to the range of the signal (\%RMSE). Consistencies were calculated for signals from start of trunk movement to gait initiation. Consistencies were considered acceptable when \%RMSE was below 10\%. A single measures, twoway mixed model, type consistency and intra-class correlation coefficient (ICC) was calculated to quantify agreement between DynaPort, Optotrak and switches.

\section{Results}

Generally, acceptable (RMSE $<10 \%$ ) differences were found between DynaPort and Optotrak based angular and linear motions of the trunk (Table 1). Exceptions were sideways displacements and non-sagittal plane rotations, where actual motions were small, resulting in RMSE of $40.1 \pm 47.4 \%$.

Agreement between DynaPort and Optotrak based temporal events and sub-phase durations was high, with ICC values of 0.988 or higher and mean absolute differences below $72 \pm 42 \mathrm{~ms}$ (Table 2).

With exception of the vertical velocity at heel-strike, agreement between DynaPort and Optotrak based trunk kinematics was high, with ICC values of 0.867 or higher. Mean absolute differences were $0.45 \pm 0.35^{\circ}$ for flexion range, $16.9 \pm 16.6^{\circ}$ for maximum

Table 1

RMSE and \%RMSE values for 3D accelerations, velocities, displacements and angles in the global coordinate system between the Optotrak and DynaPort.

\begin{tabular}{lllc}
\hline Signal & Axis & RMSE & \%RMSE \\
\hline Acceleration $\left(\mathrm{m} / \mathrm{s}^{2}\right)$ & Vertical & $0.33 \pm 0.16$ & $4.0 \pm 1.2$ \\
& Sideways & $0.23 \pm 0.13$ & $5.1 \pm 1.7$ \\
& Forward & $0.34 \pm 0.13$ & $5.8 \pm 1.9$ \\
Velocity (m/s) & Vertical & $0.04 \pm 0.02$ & $4.6 \pm 4.5$ \\
& Sideways & $0.02 \pm 0.01$ & $9.4 \pm 6.0$ \\
& Forward & $0.07 \pm 0.03$ & $6.1 \pm 3.3$ \\
Displacement (m) & Vertical & $0.02 \pm 0.02$ & $5.9 \pm 8.9$ \\
& Sideways & $0.01 \pm 0.01$ & $40.1 \pm 47.4$ \\
& Forward & $0.04 \pm 0.03$ & $6.0 \pm 4.5$ \\
Rotation $(\mathrm{deg})$ & Axial & $1.33 \pm 0.78$ & $18.1 \pm 11.3$ \\
& Flexion & $0.55 \pm 0.18$ & $1.4 \pm 0.8$ \\
& Lateral & $1.66 \pm 1.25$ & $36.5 \pm 35.4$ \\
\hline
\end{tabular}

\section{Table 2}

Mean difference, mean absolute difference and ICC for temporal events and subphases between the Optotrak or switches and DynaPort. Duration of sub-phases is displayed in seconds.

\begin{tabular}{|c|c|c|c|c|}
\hline Events/Phases & $\begin{array}{l}\text { Mean differ- } \\
\text { ence (ms) }\end{array}$ & $\begin{array}{l}\text { Mean abs dif- } \\
\text { ference (ms) }\end{array}$ & ICC & Duration (s) \\
\hline Start & $4 \pm 13$ & $8 \pm 11$ & 1.000 & - \\
\hline Seat-off & $-33 \pm 43$ & $46 \pm 28$ & 0.999 & - \\
\hline $\begin{array}{l}\text { Maximum flexion } \\
\text { angle }\end{array}$ & $3 \pm 8$ & $6 \pm 6$ & 1.000 & - \\
\hline End rising phase & $7 \pm 8$ & $7 \pm 8$ & 1.000 & - \\
\hline Heel-strike & $30 \pm 35$ & $40 \pm 22$ & 1.000 & - \\
\hline Start - heel-strike & $25 \pm 38$ & $38 \pm 25$ & 0.998 & $1.54 \pm 0.62$ \\
\hline $\begin{array}{l}\text { Start - end rising } \\
\text { phase }\end{array}$ & $3 \pm 12$ & $9 \pm 9$ & 0.999 & $1.29 \pm 0.36$ \\
\hline Seat-off - heel-strike & $65 \pm 52$ & $71 \pm 42$ & 0.996 & $1.05 \pm 0.55$ \\
\hline $\begin{array}{l}\text { Seat-off - end rising } \\
\text { phase }\end{array}$ & $40 \pm 45$ & $51 \pm 32$ & 0.988 & $0.80 \pm 0.28$ \\
\hline $\begin{array}{l}\text { Start - maximum } \\
\text { flexion angle }\end{array}$ & $-1 \pm 14$ & $10 \pm 10$ & 0.998 & $0.76 \pm 0.23$ \\
\hline $\begin{array}{l}\text { Maximum flexion } \\
\text { angle - end rising } \\
\text { phase }\end{array}$ & $4 \pm 10$ & $7 \pm 9$ & 0.999 & $0.53 \pm 0.19$ \\
\hline $\begin{array}{l}\text { End rising phase - } \\
\text { heel-strike }\end{array}$ & $22 \pm 38$ & $36 \pm 24$ & 0.991 & $0.25 \pm 0.33$ \\
\hline
\end{tabular}


Table 3

Mean difference, mean absolute difference and ICC for trunk kinematics between the Optotrak and DynaPort and corresponding mean values for the DynaPort.

\begin{tabular}{|c|c|c|c|c|c|}
\hline Trunk kinematics & Unit & Mean difference & Mean abs difference & ICC & Mean \\
\hline Flexion range & deg & $0.33 \pm 0.53$ & $0.45 \pm 0.35$ & 0.999 & $42.17 \pm 10.73$ \\
\hline Maximum flexion velocity & $\mathrm{deg} / \mathrm{s}$ & $-15,87 \pm 17.16$ & $16.90 \pm 16.59$ & 0.943 & $144.16 \pm 48.48$ \\
\hline Maximum forward velocity & $\mathrm{m} / \mathrm{s}$ & $0.06 \pm 0.10$ & $0.10 \pm 0.06$ & 0.957 & $1.30 \pm 0.33$ \\
\hline Forward velocity at seat-off & $\mathrm{m} / \mathrm{s}$ & $-0.05 \pm 0.07$ & $0.07 \pm 0.06$ & 0.867 & $0.51 \pm 0.21$ \\
\hline Forward velocity at heel-strike & $\mathrm{m} / \mathrm{s}$ & $0.04 \pm 0.11$ & $0.09 \pm 0.07$ & 0.938 & $1.25 \pm 0.28$ \\
\hline Maximum vertical velocity & $\mathrm{m} / \mathrm{s}$ & $-0.01 \pm 0.04$ & $0.03 \pm 0.02$ & 0.989 & $0.75 \pm 0.24$ \\
\hline Vertical velocity at heel-strike & $\mathrm{m} / \mathrm{s}$ & $0.03 \pm 0.10$ & $0.08 \pm 0.07$ & 0.649 & $-0.01 \pm 0.13$ \\
\hline
\end{tabular}

flexion velocity and $0.1 \pm 0.06 \mathrm{~m} / \mathrm{s}$ or lower for velocity measures (Table 3).

\section{Discussion}

This study has shown that it is possible to use a single IS for identifying temporal events, sub-phases and relevant trunk kinematics during a STW movement. In general, assessment of movements in the sagittal plane can be calculated accurately. This study used the DynaPort, but other inertial sensors are likely to provide similar results.

Reported differences between people with and people without chair rise difficulties are higher than the absolute errors observed in the current study (Buckley et al., 2008; Chen et al., 2013; Chen and Chou, 2013; Kerr et al., 2007; Kouta and Shinkoda, 2008; Van Lummel et al., 2013). E.g., the mean absolute difference of the flexion duration was $10 \pm 10 \mathrm{~ms}$, while differences of $330 \mathrm{~ms}$ between young and older adults have been reported (Van Lummel et al., 2013). This suggests the possibility of accurately differentiating between people of different functional capabilities.

A limitation of inertial sensors is the inherent difficulty to derive heading, i.e. orientation in the horizontal plane. This implies that assumptions are needed to reduce integration drift and that analyses outside the sagittal plane are difficult. While velocities were corrected for integration drift, position estimation could not be corrected for integration drift, because the exact position at the end of the movement was not known. Accelerations, velocities and displacements were corrected for heading errors assuming that there was no sideways movement on average. This method is only applicable when static starting and ending postures are known and the movement is conducted in the sagittal plane. Furthermore, trunk motion is assessed, with both the IS and reference method, at a location lower than the optimal location for tracking trunk CoM motion (Faber et al., 2009). Therefore, trunk flexion range and velocity are probably somewhat underestimated. Furthermore, no all outcome measures appeared to be normally distributed, which may have caused some bias in ICC values. Lastly, this study only used healthy older adults. The validity of the methods still needs to be tested for specific patient groups.

Overall, the results of this study support the assessment of temporal events, sub-phases and trunk kinematics of the STW with a single IS.

\section{Conflict of interest statement}

Stefan Walgaard is an employee and Rob C. van Lummel is founder and owner of McRoberts B.V. This company is the manufacturer of the DynaPort Hybrid that was used in this study.

\section{Acknowledgments}

The authors thank Encarna Micó Amigo, Sjoerd van der Smissen and Gijs van Cappelle for their support and help during the data collection and communication with the participants of this study.

\section{References}

Bernardi, M., Rosponi, A., Castellano, V., Rodio, A., Traballesi, M., Delussu, A. Marchetti, M., 2004. Determinants of sit-to-stand capability in the motor impaired elderly. J. Electromyogr. Kinesiol. 14, 401-410.

Boonstra, M.C., van der Slikke, R., Keijsers, N.L., van Lummel, R.C., de Waal Malefijt, M.C., Verdonschot, N., 2006. The accuracy of measuring the kinematics of rising from a chair with accelerometers and gyroscopes. J. Biomech. 39, 354-358.

Buckley, T.A., Pitsikoulis, C., Hass, C.J., 2008. Dynamic postural stability during sitto-walk transitions in Parkinson disease patients. Mov. Disord. 23, 1274-1280.

Chen, T., Chang, C.-C., Chou, L.-S., 2013. Sagittal plane center of mass movement strategy and joint kinetics during sit-to-walk in elderly fallers. Clin. Biomech. 28, 807-812.

Chen, T., Chou, L.-S., 2013. Altered center of mass control during sit-to-walk in elderly adults with and without history of falling. Gait Posture 38, 696-701.

Faber, G.S., Chang, C.-C., Rizun, P., Dennerlein, J.T., 2013. A novel method for assessing the 3-D orientation accuracy of inertial/magnetic sensors. J Biomech. 46, 2745-2751.

Faber, G.S., Kingma, I., Bruijn, S.M., Dieën, J.Hv, 2009. Optimal inertial sensor location for ambulatory measurement of trunk inclination. J. Biomech. 42, 2406-2409.

Giansanti, D., Maccioni, G., 2006. Physiological motion monitoring: a wearable device and adaptative algorithm for sit-to-stand timing detection. Physiol. Meas. 27, 713.

Guralnik, J.M., Simonsick, E.M., Ferrucci, L., Glynn, R.J., Berkman, L.F., Blazer, D.G., Scherr, P.A., Wallace, R.B., 1994. A short physical performance battery assessing lower extremity function: association with self-reported disability and prediction of mortality and nursing home admission. J. Gerontol. 49 M85-M85.

Janssen, W.G., Bussmann, J.B., Horemans, H.L., Stam, H.J., 2008. Validity of accelerometry in assessing the duration of the sit-to-stand movement. Med. Biol Eng. Comput. 46, 879-887.

Kerr, A., Kerr, K., 2001. Differences in the initial propulsive force between sit-towalk and sit-to-stand in healthy subjects. Physiotherapy $87,87$.

Kerr, A., Rafferty, D., Kerr, K., Durward, B., 2007. Timing phases of the sit-to-walk movement: validity of a clinical test. Gait Posture 26, 11-16.

Kouta, M., Shinkoda, K., 2008. Differences in biomechanical characteristics of sit-towalk motion between younger and elderly males dwelling in the community. J. Phys. Ther. Sci. 20, 185-189.

Kralj, A., Jaeger, R.J., Munih, M., 1990. Analysis of standing up and sitting down in humans: definitions and normative data presentation. J. Biomech. 23 $1123-1138$.

Millor, N., Lecumberri, P., Gómez, M., Martínez-Ramírez, A., Izquierdo, M., 2013. An evaluation of the 30-s chair stand test in older adults: frailty detection based on kinematic parameters from a single inertial unit. J. Neuroeng. Rehabil. 10, 86.

Moe-Nilssen, R., 1998. A new method for evaluating motor control in gait under real-life environmental conditions. Part 1: the instrument. Clin. Biomech. 13, 320-327.

Papa, E., Cappozzo, A., 2000. Sit-to-stand motor strategies investigated in ablebodied young and elderly subjects. J. Biomech. 33, 1113-1122.

Podsiadlo, D., Richardson, S., 1991. The timed "up \& go": a test of basic functional mobility for frail elderly persons. J. Am. Geriatr. Soc. 39, 142-148.

Rispens, S.M., Pijnappels, M., Schooten, K.Sv, Beek, P.J., Daffertshofer, A., Dieën, J.Hv, 2014. Consistency of gait characteristics as determined from acceleration data collected at different trunk locations. Gait Posture 40, 187-192.

Schwenk, M., Gogulla, S., Englert, S., Czempik, A., Hauer, K., 2012. Test-retest reliability and minimal detectable change of repeated sit-to-stand analysis using one body fixed sensor in geriatric patients. Physiol. Meas. 33, 1931-1946.

Van Lummel, R., Ainsworth, E., Hausdorff, J., Lindemann, U., Beek, P., Van Dieen, J. 2012. Validation of seat-off and seat-on in repeated sit-to-stand movements using a single-body-fixed sensor. Physiol. Meas. 33, 1855. 
Van Lummel, R., Ainsworth, E., Lindemann, U., Zijlstra, W., Chiari, L., Van Campen, P., Hausdorff, J., 2013. Automated approach for quantifying the repeated sit-tostand using one body fixed sensor in young and older adults. Gait Posture 38, $153-156$.

Weiss, A., Herman, T., Plotnik, M., Brozgol, M., Giladi, N., Hausdorff, J., 2011. An instrumented timed up and go: the added value of an accelerometer for identifying fall risk in idiopathic fallers. Physiol. Meas. 32, 2003.

Weiss, A., Herman, T., Plotnik, M., Brozgol, M., Maidan, I., Giladi, N., Gurevich, T., Hausdorff, J.M., 2010. Can an accelerometer enhance the utility of the timed up
\& go test when evaluating patients with Parkinson's disease? Med. Eng. Phys. $32,119-125$.

Zijlstra, A., Mancini, M., Lindemann, U., Chiari, L., Zijlstra, W., 2012. Sit-stand and stand-sit transitions in older adults and patients with Parkinson's disease: event detection based on motion sensors versus force plates. J. Neuroeng. Rehabil. 9, 75. 